\title{
Estudo da Degradação da Biomassa de Três Espécies de Plantas Aguáticas No ReservatóRIO da UHE de AMERICANA-SP ${ }^{1}$
}

\author{
Study on the Degradation of Three Aquatic Weeds at the Americana-SP Reservoir in Brazil \\ NEGRISOLI, E. ${ }^{2}$, CORREAA, M.R. ${ }^{2}$, VELINI, E.D. ${ }^{3}$, BRAVIN, L.F. ${ }^{4}$, MARCHI, S.R. ${ }^{5}$, \\ CAVENAGHI, A.L. ${ }^{2}$ e ROSSI, C.V.S. ${ }^{5}$
}

\begin{abstract}
RESUMO - O estudo de decomposição de plantas aquáticas foi realizado na UHE de AmericanaCPFL, com o objetivo de avaliar a taxa de degradação de plantas na própria água do reservatório. Foram consideradas como variáveis a profundidade (superfície, 3,5 e 7,0 m), as espécies de plantas aquáticas (Eichhornia crassipes, Brachiaria subquadripara e Pistia stratiotes) e o tipo de processamento a que estas foram submetidas (planta inteira, planta picada manualmente e com aplicação de fogo). Este estudo indicou que as espécies E. crassipes e $P$. stratiotes foram rapidamente decompostas no reservatório, ao contrário de $B$. subquadripara. Um outro fator importante observado foi a similaridade na decomposição das plantas, independentemente da profundidade testada. Essas diferenças devem ser consideradas na elaboração de planos de manejo e na previsão do impacto ambiental de programas de controle de plantas aquáticas.
\end{abstract}

Palavras-chave: macrófitas aquáticas, decomposição, água.

\begin{abstract}
A study on aquatic weed decomposition was carried out at the Americana-SP reservoir in Brazil, to compare degradation rate of the plants in the reservoir's water itself. Three variables were considered as treatments: depth (surface, 3.5 and $7.0 \mathrm{~m}$ ); aquatic weed species (Eichhornia crassipes, Brachiaria subquadripara, and Pistia stratiotes) and type of handling the aquatic weeds (whole plant, hand cut plant, and burnt plant). The results indicated that the species $\mathbf{E}$. crassipes and $\boldsymbol{P}$. stratiotes had a fast decomposition in the reservoir, unlike B. subquadripara. These differences must be considered in the elaboration of management plans and prediction of environmental impact of aquatic plant control programs. The most positive aspect of the study was to demonstrate that decomposition at $3.5 \mathrm{~m}$ and $7.0 \mathrm{~m}$ of depth had practically the same speed observed on the surface.
\end{abstract}

Keywords: aquatic macrophytes, decomposition, water.

\section{INTRODUÇÃO}

O consumo de água nas atividades humanas é muito variável no mundo. A crescente demanda de água ocasionada pelo crescimento populacional e pelo desenvolvimento industrial e agrícola tem gerado permanente pressão sobre os recursos hídricos superficiais e subterrâneos. Outro importante uso da água é na produção de hidroeletricidade, causando impactos tanto positivos como negativos (Tundisi, 2003).

Uma das principais transformações geradas pela atividade humana e de importância vital para a nossa sobrevivência é a alteração da quantidade e da qualidade de nossos recursos hídricos. Embora a água seja um

Recebido para publicação em 20.9.2005 e na forma revisada em 5.5.2006.

2 Eng.-Agr. Doutor, Departamento de Produção Vegetal - Faculdade de Ciências Agronômicas - FCA/UNESP, Caixa Postal 237, 18603-970 Botucatu-SP, Brasil, <ednegri@fca.unesp.br>; ${ }^{3}$ Prof. Adjunto, Departamento de Produção Vegetal - FCA/UNESP; ${ }^{4}$ Eng. Naval. Doutor, Departamento de Engenharia Rural - FCA/UNESP; ${ }^{5}$ Eng.-Agr. Doutorando em Agricultura do Departamento de Produção Vegetal - FCA/UNESP. 
recurso renovável, atualmente encontra-se limitado e em quantidade lentamente decrescente, se for considerado o esgotamento dos aqüíferos subterrâneos e a degradação ambiental provocada pelo desmatamento das nascentes (Salati et al., 2002).

Dentre os problemas observados, podemse destacar as infestações de algumas espécies de plantas aquáticas. Esse fato é confirmado por Marcondes et al. (2003), os quais relataram que, em certas condições, a vegetação aquática pode se tornar problemática, pois seu crescimento excessivo pode afetar os usos mais nobres da água, como irrigação, abastecimento de cidades e indústrias, navegação, recreação e geração de energia. Além disso, pode ainda haver empobrecimento do ambiente para peixes, aves e outros organismos, tendo em vista a formação de grandes infestações de uma única espécie.

Um grande dano à geração de energia por plantas aquáticas ocorreu na Usina Hidroelétrica Eng. Sousa Dias, que controla o reservatório de Jupiá, situado entre os estados de São Paulo e Mato Grosso do Sul, onde, devido ao aumento na vazão do reservatório, ocorre a fragmentação das plantas aquáticas submersas e a formação de ilhas flutuantes de taboa, aguapé e alface-d'água. Essa vegetação desloca-se pelo reservatório rumo à usina, causando sérios danos à geração de energia, pois ela se acumula nas grades de proteção da tomada d'água das unidades geradoras (CESP, 1998).

O controle de plantas aquáticas no Brasil vem sendo estudado por diversos pesquisadores: Velini (1998), Negrisoli et al. (2003), Martins et al. (2003), Guimarães et al. (2003), Carbonari et al. (2003) e Cardoso et al. (2004). No entanto, informações sobre a decomposição dessas macrófitas são escassas, podendo ser destacados os estudos de Bianchini Jr. (1985), Bianchini Jr. \& Toledo (1998) e Corrêa (2002), embora tenham fundamental importância na elaboração de planos de manejo e na previsão do impacto ambiental de programas de controle de plantas aquáticas.

Este trabalho teve como objetivo avaliar a degradação da biomassa de três espécies de plantas aquáticas, Eichhornia crassipes, Brachiaria subquadripara e Pistia stratiotes, na própria água do reservatório da UHE de Americana/CPFL-SP.

\section{MATERIAL E MÉTODOS}

O experimento foi conduzido no reservatório da UHE de Americana-SP, sendo parte das análises realizada no próprio reservatório e outra no Laboratório de Matologia do Departamento de Produção Vegetal/Agricultura, da UNESP, campus de Botucatu-SP, com início no dia 5 de maio de 2004.

Neste estudo de decomposição foram utilizadas três espécies de macrófitas predominantes no reservatório: Brachiaria subquadripara (tenner-grass), Eichhornia crassipes (aguapé) e Pistia stratiotes (alface-d'água).

As plantas estudadas foram coletadas no próprio reservatório, e os tratamentos utilizados foram: Tratamento 1 - Plantas inteiras: foram utilizadas as três espécies em estádio normal de desenvolvimento; Tratamento 2 Plantas fragmentadas: as três espécies foram fragmentadas manualmente, procurando reproduzir as condições de operação do picador que se encontrava em uso no reservatório (Figura 1); e Tratamento 3 - Plantas com aplicação de fogo: o fogo foi aplicado com o auxílio de um aplicador manual sobre as três espécies estudadas, e estas não sofreram fragmentação (Figura 2).

O delineamento estatístico utilizado foi inteiramente casualizado, sendo avaliados três tratamentos em três diferentes profundidades, com cinco repetições para cada espécie de macrófita.

As plantas foram pesadas em quantidades iniciais de $5 \mathrm{~kg}$ de matéria fresca (MF) para as espécies E. crassipes e $P$. stratiotes e $2,5 \mathrm{~kg}$ (MF) para B. subquadripara, sendo acondicionadas em sacos de polipropileno com dimensões de $47 \times 80 \mathrm{~cm}$, contendo 28 fios simples no sentido transversal e 13,5 fios duplos no sentido longitudinal, a cada $10 \mathrm{~cm}$.

Os sacos foram distribuídos em três diferentes profundidades dentro do reservatório: superfície, 3,5 e 7,0 m (Figuras 3 e 4). As avaliações constaram de pesagem periódica, aos 28 e 80 dias, e retirada de amostra do material para quantificação da matéria seca. 


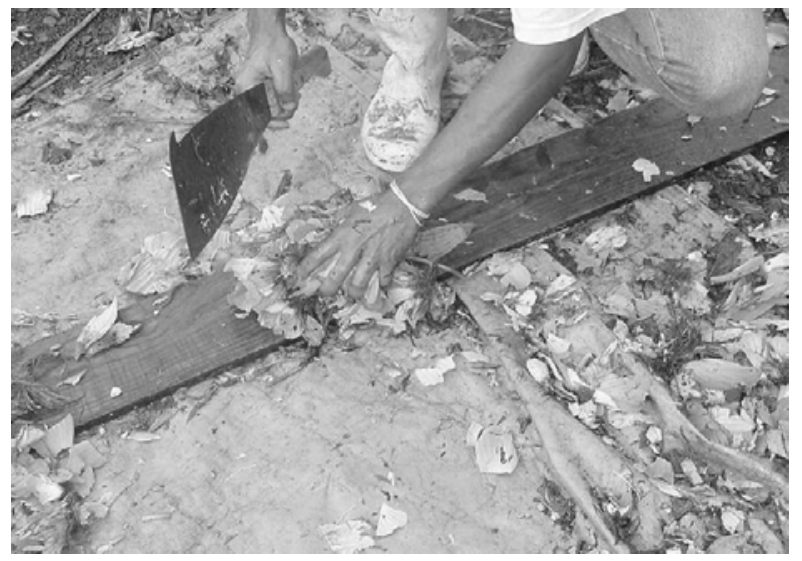

Figura 1 - Processo de fragmentação das plantas de alfaced'água.

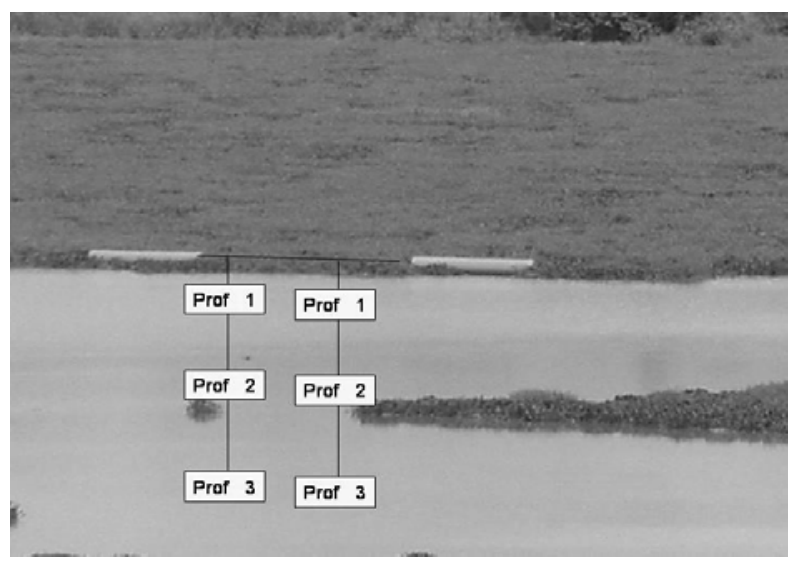

Figura 3 - Exemplo da colocação das plantas em diferentes profundidades.

Aos 28 dias após o início do experimento, foi iniciado o processo de coleta das plantas na água. Após um período de repouso das amostras, para retirada do excesso de água, estas foram pesadas, sendo em seguida coletada uma alíquota de cada repetição, para determinação da matéria seca, a fim de correlacionar com o material inicial.

Após esse processo de avaliação, a biomassa restante foi novamente conduzida ao reservatório. Esse processo foi repetido aos 80 dias para todos os tratamentos.

\section{RESULTADOS E DISCUSSÃO}

Na Tabela 1 estão apresentados os resultados das avaliações da biomassa das plantas

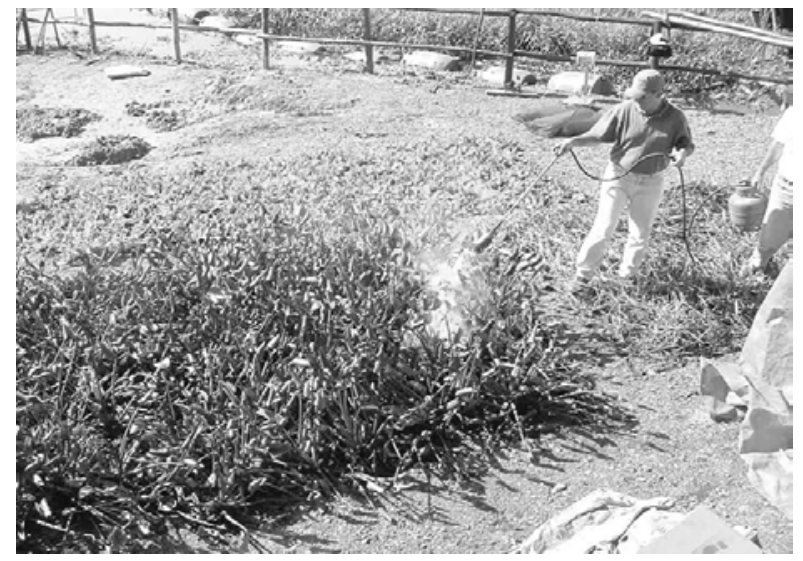

Figura 2 - Processo de tratamento com fogo em plantas de $E$. crassipes.

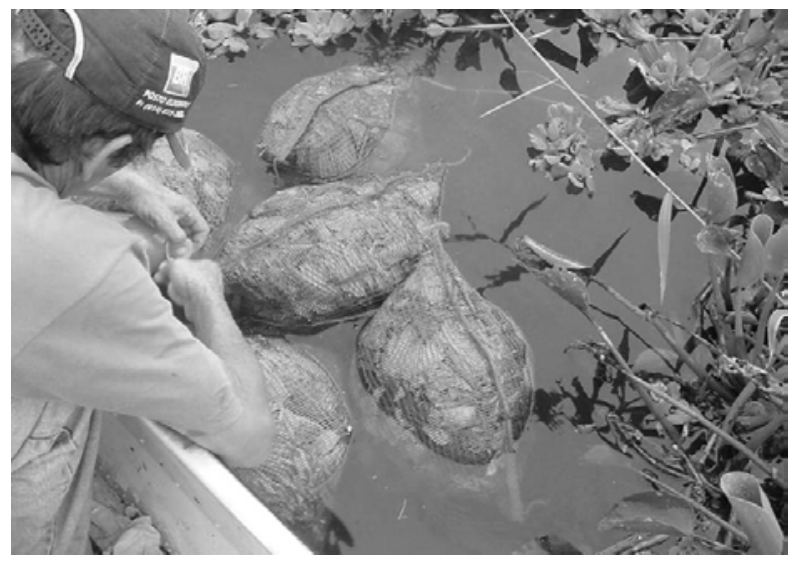

Figura 4 - Amostras de Pistia stratiotes colocadas em superfície.

de E. crassipes. Observa-se que a degradação dessa espécie foi rápida, tendo alcançado até 55\% no período inicial de 28 dias em algumas condições. Esse aspecto foi também observado por Asaeda et al. (2000), que, estudando a decomposição de macrófitas, notaram a aceleração do processo durante os primeiros 15 dias, com decréscimo gradual até o final do estudo.

Não houve efeito significativo em relação aos tratamentos com plantas inteiras e trituradas nas diferentes profundidades, mostrando similaridade em termos de velocidade de decomposição. Quando feita a comparação dos resultados encontrados para plantas tratadas com fogo, observou-se um processo degradativo significativamente inferior a uma 
Tabela 1 - Valores de degradação da biomassa, expressos em porcentagem, de Eichhornia crassipes verificados no estudo de decomposição no próprio reservatório. Americana-SP, 2004

\begin{tabular}{|c|c|c|c|c|}
\hline \multirow{2}{*}{ Avaliação } & \multirow{2}{*}{ Tratamento } & \multicolumn{3}{|c|}{ Profundidade $(\mathrm{m})$} \\
\hline & & superfície & 3,5 & 7,5 \\
\hline \multirow{3}{*}{0 dia } & Planta Inteira & - & - & - \\
\hline & Planta Picada & - & - & - \\
\hline & Tratada com Fogo & - & - & - \\
\hline F Plant. & & \multicolumn{3}{|c|}{-} \\
\hline F Prof. & & \multicolumn{3}{|c|}{-} \\
\hline F. Plant. * Prof. & & \multicolumn{3}{|c|}{-} \\
\hline $\mathrm{CV}$ & & \multicolumn{3}{|c|}{-} \\
\hline \multirow{3}{*}{28 dias } & Planta Inteira & $44,8 \mathrm{Ba}$ & $39,3 \mathrm{Ba}$ & $39,8 \mathrm{Ba}$ \\
\hline & Planta Picada & $52,6 \mathrm{ABa}$ & $49,4 \mathrm{Aa}$ & $48,0 \mathrm{ABa}$ \\
\hline & Tratada com Fogo & $55,4 \mathrm{Aa}$ & $36,9 \mathrm{Bb}$ & $51,4 \mathrm{Aa}$ \\
\hline F Plant. & & \multicolumn{3}{|c|}{5,53 ** } \\
\hline F Prof. & & \multicolumn{3}{|c|}{$5,80 * *$} \\
\hline F. Plant. * Prof. & & \multicolumn{3}{|c|}{$2,07^{\text {ns }}$} \\
\hline $\mathrm{CV}$ & & \multicolumn{3}{|c|}{14,41} \\
\hline DMS & & \multicolumn{3}{|c|}{9,782} \\
\hline \multirow{3}{*}{80 dias } & Planta Inteira & $69,3 \mathrm{Ba}$ & 71,9 Aa & $71,8 \mathrm{Aa}$ \\
\hline & Planta Picada & $79,2 \mathrm{Aa}$ & $72,1 \mathrm{Aa}$ & \begin{tabular}{l}
$49,9 \mathrm{Bb}$ \\
\hdashline,$\ldots$
\end{tabular} \\
\hline & Tratada com Fogo & $82,4 \mathrm{Aa}$ & $81,0 \mathrm{Aa}$ & $69,7 \mathrm{Aa}$ \\
\hline F Plant & & \multicolumn{3}{|c|}{$14,75 * *$} \\
\hline F Prof & & \multicolumn{3}{|c|}{$8,41 * *$} \\
\hline F. Plant. * Prof. & & \multicolumn{3}{|c|}{$6,52 * *$} \\
\hline $\mathrm{CV}$ & & \multicolumn{3}{|c|}{8,90} \\
\hline DMS & & \multicolumn{3}{|c|}{9,347} \\
\hline
\end{tabular}

** significativo a $1 \%$ de probabilidade; ${ }^{\text {ns }}$ não-significativo.

Médias seguidas de mesma letra, maiúscula na coluna e minúscula na linha, não diferem estatisticamente entre si pelo teste $\mathrm{t}(\mathrm{P}>0,05)$.

profundidade de $3,5 \mathrm{~m}$. Entretanto, a decomposição se mostrou intensa nas três profundidades e tratamentos estudados.

Na avaliação da degradação, aos 80 dias, não se observou efeito de profundidade nos tratamentos com plantas inteiras e submetidas ao fogo. No entanto, em plantas de E. crassipes trituradas, o processo degradativo foi mais intenso em menores profundidades, sendo estatisticamente superior ao obtido a 7,0 m.

Em relação à comparação feita entre os tratamentos, nota-se que a degradação da biomassa mantida na superfície foi significativamente menos intensa em plantas inteiras. Em maior profundidade, esse aspecto foi notado em plantas trituradas.

Em todas as três formas de manejo, puderam ser observadas mudanças na estrutura e coloração das plantas (Figura 5). A biomassa restante tornou-se desuniforme, com coloração escura, bem diferente do material inicial, onde podiam ser diferenciados caule, folhas e sistema radicular. Esse aspecto corrobora os dados obtidos por Bianchini Jr. et al. (1998), Cunha \& Bianchini Jr. (1998) e Pagioro \& Thomaz (1999), em que a perda de massa das macrófitas estudadas foi verificada.

Os resultados indicaram que a decomposição de E. crassipes ocorreu rapidamente no reservatório, independentemente da profundidade considerada. Se no futuro forem utilizadas práticas de controle que impliquem submersão das plantas, pode ocorrer a decomposição destas plantas sem que haja acúmulo delas no fundo do reservatório.

Guando se analisam as informações obtidas para Pistia stratiotes, observa-se que a decomposição foi bastante intensa e rápida para todos os tratamentos e profundidades avaliados (Tabela 2). As porcentagens médias de degradação ocorridas na primeira avaliação, aos 28 dias, foram de aproximadamente 80\%. Nessa avaliação, o processo de degradação mostrou-se intenso tanto para os tratamentos como para profundidade. Comparando-se os tratamentos, foi possivel observar que a porcentagem de decomposição foi maior para as plantas que sofreram trituração, independentemente da profundidade analisada. $O$ fator profundidade não foi limitante para esta espécie durante esse primeiro período avaliado, exceto para plantas inteiras dispostas na superfície, com índice significativamente inferior.

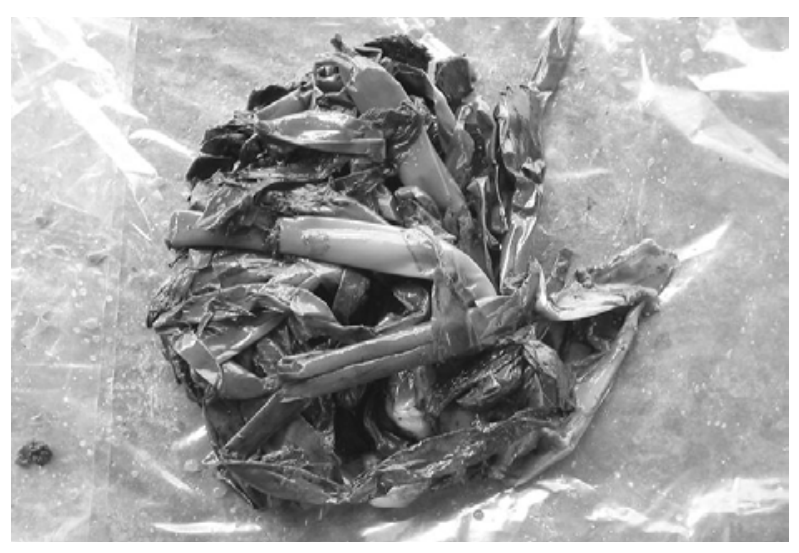

Figura 5 - Aspecto de plantas de E. crassipes após a primeira avaliação (28 dias). 
Tabela 2 - Valores de degradação da biomassa, expressos em porcentagem, de Pistia stratiotes verificados no estudo de decomposição no próprio reservatório. Americana-SP, 2004

\begin{tabular}{|c|c|c|c|c|}
\hline \multirow{2}{*}{ Avaliação } & \multirow{2}{*}{ Tratamento } & \multicolumn{3}{|c|}{ Profundidade $(\mathrm{m})$} \\
\hline & & superfície & 3,5 & 7,5 \\
\hline \multirow{3}{*}{0 dia } & Planta Inteira & - & - & - \\
\hline & Planta Picada & - & - & - \\
\hline & Tratada com Fogo & - & - & - \\
\hline F Plant. & & \multicolumn{3}{|c|}{-} \\
\hline F Prof. & & \multicolumn{3}{|c|}{-} \\
\hline F. Plant. * Prof. & & \multicolumn{3}{|c|}{-} \\
\hline $\mathrm{CV}$ & & \multicolumn{3}{|c|}{-} \\
\hline \multirow{3}{*}{28 dias } & Planta Inteira & $42,1 \mathrm{Cb}$ & $81,3 \mathrm{Aa}$ & $78,4 \mathrm{Ba}$ \\
\hline & Planta Picada & $81,7 \mathrm{Aa}$ & $81,2 \mathrm{Aa}$ & $83,4 \mathrm{Aa}$ \\
\hline & Tratada com Fogo & $77,7 \mathrm{Ba}$ & $73,3 \mathrm{Bb}$ & $75,5 \mathrm{Ca}$ \\
\hline F Plant. & & \multicolumn{3}{|c|}{$2,56^{*}$} \\
\hline F Prof. & & \multicolumn{3}{|c|}{$30,93 * *$} \\
\hline F. Plant. * Prof. & & \multicolumn{3}{|c|}{$3,02 * *$} \\
\hline $\mathrm{CV}$ & & \multicolumn{3}{|c|}{2,71} \\
\hline DMS & & \multicolumn{3}{|c|}{3,144} \\
\hline \multirow{3}{*}{80 dias } & Planta Inteira & 91,9 Aa & $91,8 \mathrm{Aa}$ & $91,2 \mathrm{Aa}$ \\
\hline & Planta Picada & $90,1 \mathrm{Aa}$ & $94,1 \mathrm{Aa}$ & $94,1 \mathrm{Aa}$ \\
\hline & Tratada com Fogo & $94,3 \mathrm{Aa}$ & $82,4 \mathrm{Bb}$ & $90,5 \mathrm{Aa}$ \\
\hline F Plant & & \multicolumn{3}{|c|}{$2,61 *$} \\
\hline F Prof & & \multicolumn{3}{|c|}{$4,04 * *$} \\
\hline F. Plant. * Prof. & & \multicolumn{3}{|c|}{$6,78 * *$} \\
\hline $\mathrm{CV}$ & & \multicolumn{3}{|c|}{3,56} \\
\hline DMS & & \multicolumn{3}{|c|}{4,743} \\
\hline
\end{tabular}

** significativo a $1 \%$ de probabilidade; ${ }^{\text {ns }}$ não-significativo.

Médias seguidas de mesma letra, maiúscula na coluna e minúscula na linha, não diferem estatisticamente entre si pelo teste $t(P>0,05)$.

Na segunda avaliação, aos 80 dias após o início do estudo, embora o processo degradativo tenha ocorrido com menor velocidade, as porcentagens médias de decomposição chegaram a 94\%. Fellerhoff et al. (2003) também constataram reduções rápidas da matéria seca de algumas macrófitas tropicais, em torno de 60 a $90 \%$, nas três primeiras semanas de estudos. No entanto, seu estudo foi realizado em mesocosmos, onde alguns fatores, como concentração de oxigênio e temperatura, foram determinantes.

Não foi observado nesse período efeito significativo entre os tratamentos e as profundidades, com exceção para plantas submetidas ao fogo e mantidas à profundidade de $3,5 \mathrm{~m}$, em que se observou menor porcentagem de degradação.
Igualmente ao observado para a espécie E. crassipes, verificou-se drástica alteração da estrutura e forma das plantas de P. stratiotes, caracterizada pela grande diminuição do volume das amostras e mudança na coloração. Estas ficaram com aspecto de massa e coloração verde-escura, além de exalarem forte odor em todos os tratamentos testados (Figura 6).

A análise de todos os resultados obtidos para $P$. stratiotes indica que a sua decomposição no reservatório é ainda mais rápida do que a de E. crassipes. Há conseqüências positivas e negativas a serem consideradas. Se por um lado a rápida decomposição é uma garantia de que as plantas controladas no reservatório serão rapidamente eliminadas do sistema, a rápida decomposição preocupa, por demandar maiores quantidades de oxigênio nas fases imediatamente posteriores ao controle.

Os resultados referentes à decomposição de Brachiaria subquadripara estão apresentados na Tabela 3, demonstrando que esta espécie apresentou as menores velocidades de decomposição.

A porcentagem média de decomposição na primeira avaliação (28 dias) foi de aproximadamente $27 \%$, com máxima de $33 \%$, não sendo observado efeito significativo na porcentagem de degradação para tratamento ou profundidade. Em relação aos tratamentos, as plantas submetidas ao fogo apresentaram maior porcentagem de degradação, sendo a menor porcentagem encontrada em plantas mantidas inteiras, em todas as profundidades avaliadas.

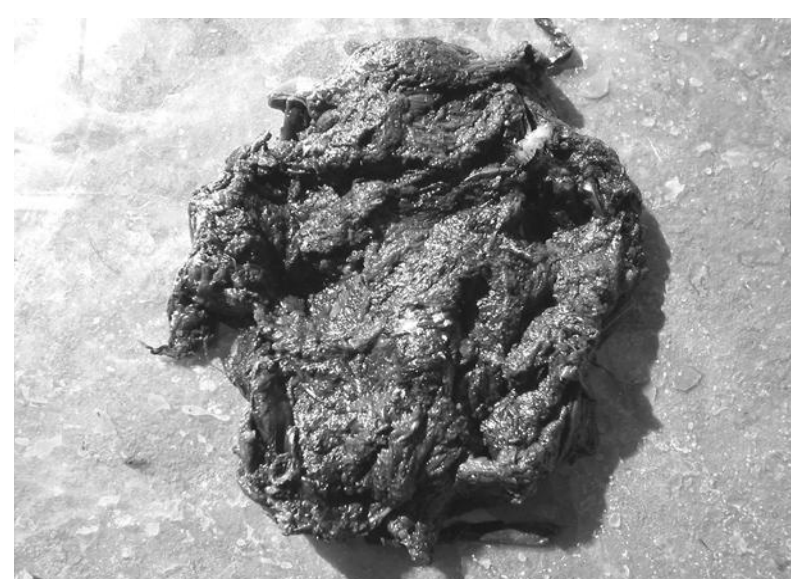

Figura 6 - Aspecto de plantas de P. stratiotes durante a primeira avaliação (28 dias). 
Tabela 3 - Valores de degradação da biomassa, expressos em porcentagem, de Brachiaria subquadripara verificados no estudo de decomposição no próprio reservatório. Americana-SP, 2004

\begin{tabular}{|c|c|c|c|c|}
\hline \multirow{2}{*}{ Avaliação } & \multirow{2}{*}{ Tratamento } & \multicolumn{3}{|c|}{ Profundidade (m) } \\
\hline & & \begin{tabular}{|l|} 
superfície \\
\end{tabular} & 3,5 & 7,5 \\
\hline \multirow{3}{*}{0 dia } & Planta Inteira & - & - & - \\
\hline & Planta Picada & - & - & - \\
\hline & Tratada com Fogo & - & - & - \\
\hline F Plant. & & \multicolumn{3}{|c|}{-} \\
\hline F Prof. & & \multicolumn{3}{|c|}{-} \\
\hline F. Plant. * Prof. & & \multicolumn{3}{|c|}{-} \\
\hline $\mathrm{CV}$ & & \multicolumn{3}{|c|}{-} \\
\hline \multirow{3}{*}{28 dias } & Planta Inteira & 18,7 & 21,4 & 12,3 \\
\hline & Planta Picada & 25,6 & 33,6 & 24,7 \\
\hline & Tratada com Fogo & 33,1 & 33,4 & 32,6 \\
\hline F Plant. & & \multicolumn{3}{|c|}{$4,95 * *$} \\
\hline F Prof. & & \multicolumn{3}{|c|}{$7,64 * *$} \\
\hline F. Plant. * Prof. & & \multicolumn{3}{|c|}{$1,51^{\mathrm{ns}}$} \\
\hline $\mathrm{CV}$ & & \multicolumn{3}{|c|}{28,32} \\
\hline DMS & & \multicolumn{3}{|c|}{-} \\
\hline \multirow{3}{*}{80 dias } & Planta Inteira & 59,7 & 37,3 & 47,2 \\
\hline & Planta Picada & 43,2 & 34,8 & 25,7 \\
\hline & Tratada com Fogo & 45,1 & 45,2 & 43,4 \\
\hline F Plant & & \multicolumn{3}{|c|}{$1,86^{\mathrm{ns}}$} \\
\hline F Prof & & \multicolumn{3}{|c|}{$2,54 *$} \\
\hline F. Plant. * Prof. & & \multicolumn{3}{|c|}{$0,82^{\text {ns }}$} \\
\hline $\mathrm{CV}$ & & \multicolumn{3}{|c|}{36,03} \\
\hline DMS & & \multicolumn{3}{|c|}{-} \\
\hline
\end{tabular}

** significativo a $1 \%$ de probabilidade, ${ }^{\text {ns }}$ não-significativo.

Médias seguidas de mesma letra, maiúscula na coluna e minúscula na linha, não diferem estatisticamente entre si pelo teste $t(P>0,05)$.

Na segunda avaliação, realizada aos 80 dias, a taxa média de degradação foi de $43 \%$, atingindo porcentagem máxima de $59,7 \%$. Ao contrário do que foi observado no primeiro período de avaliação, maior porcentagem de decomposição foi encontrada em plantas inteiras na superfície e a 7,0 m. A uma profundidade média, de $3,5 \mathrm{~m}$, o mesmo foi obtido em plantas tratadas com fogo.

Em contraste com o verificado para as demais espécies estudadas, essa gramínea não apresentou alterações de forma durante o período de avaliação da decomposição. No entanto, ocorreu alteração na coloração da biomassa e quase completa decomposição apenas das folhas (Figura 7).

A menor taxa de decomposição gera preocupação quanto à persistência da biomassa

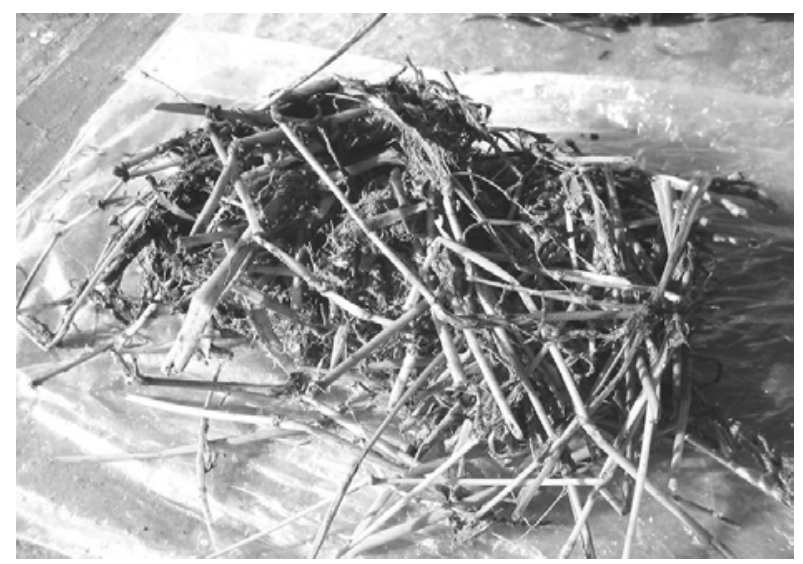

Figura 7 - Aspecto de plantas de B. subquadripara durante a primeira avaliação (28 dias).

da planta após ações de controle (químico ou por fogo), mas é positiva, uma vez que indica menor consumo de oxigênio nos períodos imediatamente subseqüentes ao controle.

Este estudo indicou que as espécies $E$. crassipes e $P$. stratiotes foram rapidamente decompostas no reservatório, ao contrário de B. subquadripara. Essas diferenças devem ser consideradas na elaboração de planos de manejo e na previsão do impacto ambiental de programas de controle de plantas aquáticas. Provavelmente, esse fator relaciona-se com o alto teor de lignina encontrado nesta espécie de graminea.

Um fator importante observado no estudo foi a similaridade na decomposição das plantas, independentemente da profundidade testada. Essa informação é de grande relevância, pois o uso de herbicidas pode promover a submersão de plantas.

\section{AGRADECIMENTOS}

À CPFL, Companhia Paulista de Força e Luz, pelo suporte técnico e financeiro. Este trabalho foi financiado pela linha de $\mathrm{P} \& \mathrm{D}$ da ANEEL.

\section{LITERATURA CITADA}

ASAEDA, T.; TRUNG, V. K.; MANATUNGE, J. Modeling the effects of macrophyte growth and decomposition on the nutrient budget in Sallow Lakes. Aquatic Bot., v. 68, p. 217-237, 2000. 
BIANCHINI JR., I. Estudo dos processos de humificação de Nymphoides indica (L) O. Kruntze. 1985. 285 f. Tese (Doutorado em Ecologia e Recursos Naturais) - Universidade Federal de São Carlos, São Carlos, 1985.

BIANCHINI JR., I. et al. Degradação anaeróbia de macrófitas aquáticas: metanogênese. In: SIMPÓSIO NACIONAL FERMENTAÇÕES - SINAFERM, 12., 1998, Uberlândia. Anais... Uberlândia: 1998. CD-ROM.

BIANCHINI, JR., I.; TOLEDO, A. P. P. Estudo da mineralização de Nymphoides indica (L) O. Kruntze. In: SEMINÁRIO REGIONAL DE ECOLOGIA, 8., 1998, São Carlos. Anais... São Pedro: Universidade Federal de Santa Catarina, 1998. v. 3. p. 1315-1329.

CARBONARI, C. A.; MARTINS, D.; TERRA, M. A. Controle de Brachiaria subquadripara e Brachiaria mutica através de diferentes herbicidas aplicados em pós-emergência. Planta Daninha, v. 21, p. 79-84, 2003. Edição especial.

CARDOSO, L. R. et al. Variabilidade genética entre populações de Pistia stratiotes L. In: CONGRESSO BRASILEIRO DA CIÊNCIA DAS PLANTAS DANINHAS, 24., 2004, São Pedro. Anais... São Pedro: SBCPD, 2004. p. 107.

COMPANHIA ENERGÉTICA DE SÃO PAULO - CESP. Conservação e manejo nos reservatórios: limnologia, ictiologia e pesca. São Paulo: 1998. 166 p.

CORRÊA, M. R. Composição química, bromatológica e decomposição de Egeria densa, Egeria najas e Ceratophyllum demersun coletadas no reservatório de Jupiá. 2002. 101 f. Dissertação (Mestrado em Agricultura) - Universidade Estadual Paulista, Botucatu, 2002.

CUNHA, M. B; BIANCHINI JR., I. Mineralização aeróbia de Cabomba piauhyensis e Scirpus cubensis. Acta Limn. Bras., v. 10, n. 1, p. 81-91, 1998.

FELLERHOFF, C.; VOSS, M.; WANTZEN, K. M. Stable carbon and nitrogen isotope signatures of decomposing tropical macrophytes. Aquatic Ecol., v. 37, p. 361-375, 2003.
GUIMARÃES, G. L. et al. Metodologia para avaliação de impacto ambiental de macrófitas em mesocosmos. Planta Daninha, v. 21, p. 37-42, 2003. Edição especial.

MARCONDES, D. A. S.; MUSTAFÁ, A. F.; TANAKA, R. H. Estudos para manejo integrado de plantas aquáticas no reservatório de Jupiá. In: THOMAZ, M. S.; BINI, M. L. Ecologia e manejo de macrófitas aquáticas. Maringá: EDUEM, 2003. v. 1. p. 299-318.

MARTINS, D. et al. Caracterização genética de acesso de Egeria (Egeria spp.) coletados no estado de São Paulo utilizando RAPD. Planta Daninha, v. 21, p. 1-7, 2003. Edição especial.

NEGRISOLI, E. et al. Uso de diferentes herbicidas no controle de Myriophyllum aquaticum. Planta Daninha, v. 21, p. 89-92, 2003. Edição especial.

PAGIORO, T. A.; THOMAZ, S. M. Influence of the decomposition of Eichhornia azurea on selected abiotic limnological variables of different environments of the floodplain of the high Parana river. Acta Limn. Bras., v. 11, n. 2, p. 157-171, 1999.

SALATI, E.; LEMOS, H. M.; SALATI, E. Água e o desenvolvimento sustentável. In: REBOUÇAS, A. C.; BRAGA, B.; TUNDISI, J. G. (Orgs.). Águas doces no Brasil: capital ecológico, uso e conservação. 2.ed. São Paulo: Escrituras, 2002. p. 39-64.

TUNDISI, J. G. Água no século XXI; enfrentando a escassez. São Carlos: Rima Artes e Textos, 2003. v. 1. $248 \mathrm{p}$.

VELINI, E. D. Controle mecânico de plantas aquáticas no Brasil. In: WORKSHOP CONTROLE DE PLANTAS AQUÁTICAS, 1998, Brasília. Resumos... Brasília: IBAMA, 1998. p. 32-35. 